14

\title{
Взаимодействие низкоэнергетических электронов с молекулами фруктозы
}

\author{
() И.В. Чернышова, Е.Э. Контрош, П.П. Маркуш, О.Б. Шпеник
}

Институт электронной фризики НАН Украины, Ужгород, Украина

E-mail: irinav.chernyshova@gmail.com

Поступило в Редакцию 8 ноября 2016 г.

С использованием гипоциклоидального электронного спектрометра выполнены исследования взаимодействия низкоэнергетических $(0-8.50 \mathrm{eV})$ электронов с молекулами фруктозы: рассеяния и диссоциативного прилипания электронов. Результаты исследований показали, что фрагментация молекул фруктозы эффективно происходит уже при энергии электронов, близкой к нулю. В полном сечении рассеяния электронов молекулами впервые обнаружены резонансные особенности (при энергиях $\sim 3.10$ и $\sim 5.00 \mathrm{eV}$ ), находящиеся вблизи порогов образования легких ионных фрагментов $\mathrm{OH}^{-}$и $\mathrm{H}^{-}$. Проанализирована корреляция особенностей, наблюдаемых в сечениях рассеяния и диссоциативного прилипания электрона.

DOI: $10.21883 /$ PJTF.2017.22.45254.16561

Известно, что радиоактивное излучение, проходящее через биологическую материю, приводит к появлению вторичных медленных электронов, разрушающих ее $[1]$. В последнее время появилось значительное число работ, связанных с исследованием взаимодействия медленных электронов с компонентами ДНК в газовой фазе [2]. Интерес к таким исследованиям обусловлен попыткой объяснить на молекулярном уровне механизмы разрушения ДНК медленными электронами. Одной из составных частей молекулы ДНК является молекула сахара - 2-дезоксирибоза. Это объясняет интерес к изучению 
столкновений медленных электронов с молекулами сахаров и близких к ним молекул [3]. Проведенные недавно исследования диссоциативного прилипания низкоэнергетических электронов к циклическим молекулам: фурану, тетрагидрофурану и фруктозе [4] - показали, что для молекул фурана и тетрагидрофурана указанный процесс при энергиях, близких к нулю, является неэффективным, а их фрагментация происходит преимущественно через относительно высоколежащее резонансное состояние (коротоживущее состояние отрицательного иона) выше $5.00 \mathrm{eV}$. Напротив, фрагментация молекул фруктозы происходит интенсивно при энергиях налетающих электронов, близких к нулю (см. также [5]). Однако до сих пор отсутствуют данные о сечениях рассеяния медленных электронов молекулами фруктозы в области энергий 0-10 eV. Такие сечения помимо данных об упругих и неупругих столкновениях содержат информацию о резонансах, играющих важную роль в различных процессах, происходящих при столкновении медленных электронов с молекулами.

В настоящей работе приводятся результаты исследований полного сечения образования отрицательных ионов (сечения диссоциативного прилипания электронов к молекуле) и полного сечения рассеяния низкоэнергетических электронов молекулами фруктозы. Целью данных исследований было проследить корреляцию особенностей, наблюдаемых в этих сечениях.

Для изучения соударений электронов с молекулами фруктозы в газовой фазе использовался гипоциклоидальный электронный спектрометр [6], состоящий из двух идентичных гипоциклоидальных электронных монохроматоров (ГЭМ) [7], размещенных последовательно: один из них служил монохроматором электронов, а другой - анализатором рассеянных электронов. Между ними располагалась паронаполненная ячейка, служащая камерой столкновений. Выбор данного спектрометра обусловлен тем, что он может работать в области очень малых (практически нулевых) значений энергии и при этом обладает высокой степенью пропускания (до 95\%). В данных измерениях наилучшее энергетическое разрешение ГЭМ (полная ширина на полувысоте максимума продифференцированного начального участка вольт-амперной характеристики электронного тока) составляло $\Delta E_{1 / 2}=80 \mathrm{meV}$ при токе электронов $120 \mathrm{nA}$. Магнитное поле, необходимое для работы спектрометра, создавалось парой колец Гельмгольца диаметром $210 \mathrm{~mm}$.

Письма в ЖТФ, 2017, том 43, вып. 22 
Мишень в газовой фазе формировалась в резервуаре молекулярного источника, который представлял собой полый цилиндр из нержавеющей стали, резистивно нагреваемый до температуры, при которой еще не наблюдаются структурные изменения молекулы. Температуры резервуара и ячейки контролировались терморезисторами РТ-100.

Порошок фруктозы, помещенный в кварцевую ампулу, находящуюся в резервуаре молекулярного источника, постепенно нагревался до необходимой температуры. Пары фруктозы по фторопластовой трубке поступали непосредственно в паронаполняемую ячейку. Отметим, что для предотвращения конденсации молекул фруктозы на электродах спектрометра его температура поддерживалась на $20-30^{\circ} \mathrm{C}$ выше температуры резервуара с исследуемым веществом. Рабочее давление в вакуумной камере было не хуже $2 \cdot 10^{-4} \mathrm{~Pa}$.

При изучении полного сечения рассеяния электронов нами был использован традиционный - трансмиссионный - метод, в котором измерялось ослабление электронного пучка при его прохождении через пары исследуемых молекул и с использованием формулы Ламберта-Бера определялось сечение рассеяния

$$
\sigma(E)=1 / n \ell \cdot \ln \left[I_{0}(E) / I(E)\right]
$$

где $I(E)$ и $I_{0}(E)$ - интенсивности электронного пучка при наличии и в отсутствии паров мишени в камере столкновений длиной $\ell$ соответственно $n$ - плотность паров мишени.

При изучении сечения образования отрицательных ионов ток ионов, образовавшихся при соударении медленных электронов с молекулами фруктозы, детектировался коллектором ионов, который был установлен внутри камеры столкновений перпендикулярно направлению электронного пучка. Перед коллектором размещалась сетка (прозрачностью 80\%) из немагнитного материала для его защиты от попадания вторичных и рассеянных электронов. Для регистрации отрицательных ионов на коллектор подавался небольшой положительный потенциал.

Молекула фруктозы $\left(\mathrm{C}_{6} \mathrm{H}_{12} \mathrm{O}_{6}\right)-$ моносахарид, в живых организмах присутствует исключительно как $D$-изомер. Как большинство сахаров, фруктоза может существовать как ациклический линейный изомер с карбонильной группой у $\mathrm{C}_{2}$, а также в виде шести- (пиранозная форма) (вставка на рис. 1) и пятичленных колец (фуранозная форма). Каждый из этих изомеров может иметь много различных конформеров. Как

Письма в ЖТФ, 2017, том 43, вып. 22 


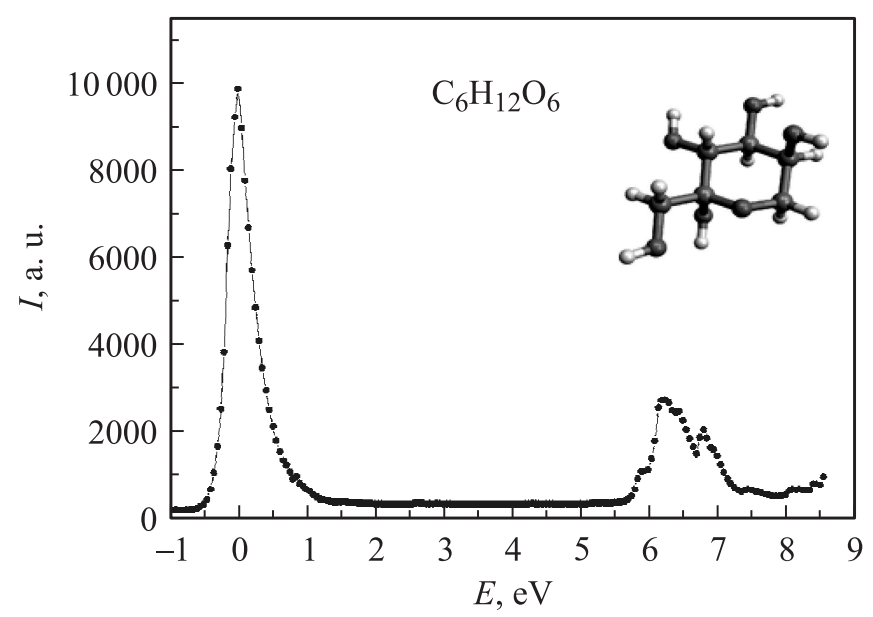

Рис. 1. Сечение диссоциативного прилипания электронов к молекуле фруктозы.

показано в [5], в газовой фазе изомерный состав будет различным с доминированием молекул пиранозной формы. В наших экспериментах использовался образец в виде кристаллического порошка белого цвета (чистота 99\%), хорошо растворимого в воде. Проведенные предварительно дериватографические исследования позволили определить температуру плавления данного образца, равную $100^{\circ} \mathrm{C}$.

На рис. 1 приведена энергетическая зависимость сечения диссоциативного прилипания электронов к молекуле фруктозы в области энергий бомбардирующих электронов $0-8.50 \mathrm{eV}$. Отметим, что измеряемое нами сечение имеет смысл полного сечения диссоциативного прилипания, поскольку измеряются токи всех образованных в камеpe столкновений отрицательных ионов (как целой молекулы, так и ее ионных фрагментов). Видно, что на энергетической зависимости сечения диссоциативного прилипания наблюдаются две выраженные структуры: первая в области энергий от 0 до $1.00 \mathrm{eV}$, вторая в области от $5.50 \mathrm{eV}$ и выше. Интенсивность второй структуры составляет приблизительно одну треть от интенсивности первого пика. Как показали результаты масс-спектрометрических исследований [4], в области энергий $0-1.00 \mathrm{eV}$ происходит образование крупных ионных 


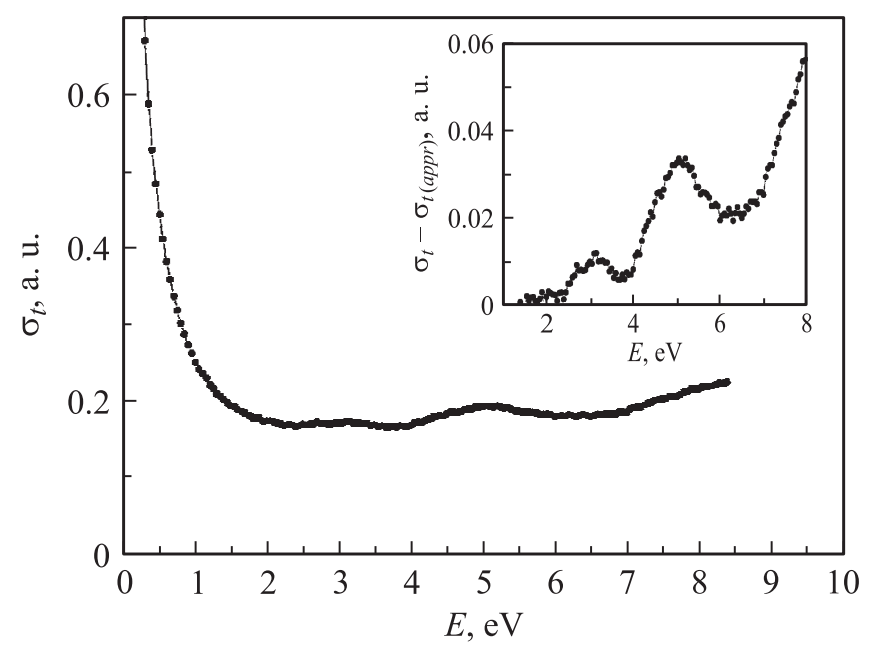

Рис. 2. Полное сечение рассеяния электронов молекулами фруктозы.

фрагментов с элиминированием одной, двух и трех молекул воды, a также ионов $\mathrm{C}_{5} \mathrm{H}_{8} \mathrm{O}_{4}^{-}, \mathrm{C}_{5} \mathrm{H}_{6} \mathrm{O}_{3}^{-}, \mathrm{C}_{3} \mathrm{H}_{4} \mathrm{O}_{2}^{-}$и $\mathrm{HCOO}^{-}$. Согласно [5], фрагментация молекулы происходит через так называемый doorway-механизм.

Выше энергии $5.50 \mathrm{eV}$ фрагментация молекулы происходит, по-видимому, через высоколежащее резонансное состояние Фешбаха с возбуждением остова молекулы (core-excited type) [5]. Для идентификации наблюдаемых нами особенностей мы воспользовались данными [4]. Сравнение наших результатов с данными этой работы показало, что наблюдаемые в указанной области энергий особенности обусловлены вкладами в полное сечение от образования легких ионных фрагментов $\mathrm{H}^{-}, \mathrm{O}^{-}$и $\mathrm{OH}^{-}$.

Энергетическая зависимость полного сечения рассеяния электронов молекулами фруктозы, измеренная в области энергий бомбардирующих электронов от 0 до $8.50 \mathrm{eV}$, приведена на рис. 2. Отличительной особенностью измеренного нами сечения является его быстрый (примерно в 5 раз) спад в диапазоне энергий бомбардирующих электронов от 0 до $1.00 \mathrm{eV}$.

Письма в ЖТФ, 2017, том 43, вып. 22 
В области энергий $1-6.00 \mathrm{eV}$ в полном сечении рассеяния электронов молекулами фруктозы наблюдаются две особенности. Эти особенности обусловлены влиянием резонансов, которые образуются при кратковременном $\left(10^{-15} \mathrm{~s}\right)$ захвате электрона молекулой. Для выделения этих резонансных особенностей и точного определения их энергетического положения, мы вычли из измеренной энергетической зависимости сечения $\left(\sigma_{t}\right)$ аппроксимированный вклад прямого (потенциального) рассеяния $\left(\sigma_{t(a p p r)}\right)[6]$. Выделенные резонансные вклады $\sigma_{t}-\sigma_{t(\text { appr })}$ показаны на вставке к рис. 2 и характеризуются максимумами при энергиях $\sim 3.10$ и $\sim 4.95 \mathrm{eV}$. Первый из них представляет собой результат перекрывания по меньшей мере двух особенностей с максимумами при 2.68 и $3.15 \mathrm{eV}$. Положение первого максимума $(2.68 \mathrm{eV})$ согласуется с порогом появления ионов $\mathrm{OH}^{-}(2.77 \mathrm{eV})$, a второго $(3.15 \mathrm{eV})$ - с порогом появления ионов $\mathrm{H}^{-}(3.25 \mathrm{eV})$, определенных в [4]. Появление максимума при $5.20 \mathrm{eV}$ может быть связано как с формированием отрицательных ионов $\mathrm{O}^{-}$[4], так и с электронным возбуждением молекулы фруктозы. Отметим, что ранее в этой области энергий наблюдалось возбуждение молекулы фурана [8], которая представляет собой пятичленный кислородсодержащий гетероцикл.

Проведенные исследования показали, что столкновения низкоэнергетических электронов с молекулами фруктозы приводят к деструктивным процессам. Разрушающее воздействие электронов начинается практически с нулевых энергий. Выше энергии $5.00 \mathrm{eV}$ столкновения с медленными электронами приводят к появлению легких анионных фрагментов, что связано с разрушением гетероциклов молекулы. Сравнение сечения диссоциативного прилипания с полным сечением рассеяния подтвердило, что выше энергии $4.00 \mathrm{eV}$ образование отрицательных ионных фрагментов носит резонансный характер.

\section{Список литературы}

[1] Boudaiffa B., Cloutier P., Hunting D., Huels M.A., Sanche L. // Science. 2000. V. 287. P. 1658.

[2] Sanche L. // Eur. Phys. J. D. 2005. V. 35. P. 367.

[3] Fujita T., Kondo M., Takayanagi T. // Comput. Theor. Chem. 2016. V. 1075. P. 70.

[4] Sulzer P., Ptasinska S., Zappa F. et al. // J. Chem. Phys. 2006. V. 125. P. 044304. 
[5] Sommerfeld T. // J. Chem. Phys. 2007. V. 126. P. 124301.

[6] Kontros J.E., Szoter L., Chernyshova I.V., Shpenik O.B. // J. Phys. B. 2002. V. 35. P. 2195.

[7] Romanyuk M.I. // Meas. Sci. Technol. 1994. V. 5. P. 912.

[8] Flicker W., Mosher O.A., Kuppermann A. // J. Chem. Phys. 1976. V. 64. P. 1315.

Письма в ЖТФ, 2017, том 43, вып. 22 